\title{
Identifying Behavioral Novelty
}

\author{
Rachael L. Brown
}

Received: 10 September 2013/Accepted: 4 November 2013/Published online: 5 December 2013

(c) Konrad Lorenz Institute for Evolution and Cognition Research 2013

\begin{abstract}
Although there is no in-principle impediment to an EvoDevo of behavior, such an endeavor is not as straightforward as one might think; many of the key terms and concepts used in EvoDevo are tailored to suit its traditional focus on morphology, and are consequently difficult to apply to behavior. In this light, the application of the EvoDevo conceptual toolkit to the behavioral domain requires the establishment of a set of tractable concepts that are readily applicable to behavioral characters. Here, I begin the type of theoretical work that needs to be undertaken in order to achieve this, focusing in particular on the key concept of "novelty." Building on existing criteria used for the identification of behavioral homology from behavioral ecology, I develop a set of operational criteria for identifying novelty in the behavioral domain. These criteria provide a conceptual foundation for the study of novelty in behavioral traits.
\end{abstract}

Keywords Behavior - EvoDevo · Homology ·

Non-homology $\cdot$ Novelty

Many of the standing definitions of "novelty" within EvoDevo have their origins in Müller (1990, pp. 99-101), who defines it as the appearance of a qualitatively new structural feature. While Müller's definition is not in error-the vast majority of work in EvoDevo concerns morphological evolution, and the definition has currency in this domain - it is not immediately clear how a notion of novelty that relies on structural similarities and differences

R. L. Brown ( $\square)$

Rotman Institute of Philosophy, Department of Philosophy,

Western University, London, ON, Canada

e-mail: rachael@rachaelbrown.net could be applied to behavioral characters. This presents a challenge for those interested in understanding behavioral novelty and innovation within the EvoDevo context (e.g., Bertossa 2011; Brown 2014).

That a novelty concept can be applied to behavior at all is prima facie motivated by some classic studies in ethology using variation in behavioral traits over time to build phylogenies. Van Tets (1965), for example, used interspecies variation in the display behaviors of the Pelicaniform birds to build a phylogeny of these species that has ultimately been largely vindicated by modern molecular evidence (Kennedy et al. 1996). More recently, McLennan et al. (1988) and Johnston and Page (1992) used fish nestassociated behaviors and reproductive strategies respectively to build phylogenies. In addition to this phylogenetic work, attempts to operationalize the concept of "innovation" in animal behavior (e.g., Reader and Laland 2003; Ramsey et al. 2007) highlight the potential for successful criteria for the identification of behavioral novelty; innovation and novelty being closely related concepts.

In this article, I build on this existing work to offer a clear account of what novelty amounts to in the behavioral domain. I begin by highlighting the centrality of the project of explaining novelty within the EvoDevo research program and outlining the prevailing approaches to novelty therein. Following this, I move on to the question of the appropriate definition of novelty for the behavioral domain.

\section{Some Background to Novelty in EvoDevo}

\section{The Origination Problem}

A key motivation of EvoDevo as a research program arises from the understanding that Darwin's theory of evolution 
by natural selection, while capable of explaining adaptation and diversity in the tree of life, cannot account for the origination of traits. More specifically, the theory of evolution by natural selection does not tell us how the supply of phenotypic variation within populations arises (i.e., how traits originate), and consequently cannot explain (a) why selection is able to occur, and (b) why certain traits exist in populations and others do not (Sterelny and Griffiths 1999, p. 384). According to proponents of EvoDevo, the received view of evolution - the Modern Synthesis (MS henceforth) - also fails to adequately respond to the question of origination, because it includes untenable empirical assumptions about the source of phenotypic variation to natural selection and the nature of the variation itself (Müller and Newman 2005; Müller 2007). ${ }^{1}$

First, it is assumed in the MS that the supply of phenotypic variation to natural selection is solely a product of unbiased genetic mutation and recombination (Dobzhansky 1957, 1971; Mayr and Provine 1981). ${ }^{2}$ The assumption of isotropism is important. With this, the phenotypic variation to selection becomes explanatorily impotent with respect to the particular outcomes of the evolutionary process over long timescales (over and above its role in providing the raw material on which selection acts). It is natural selection (rather than the nature of the supply of phenotypic variation to selection) that explains why we have the traits we have in populations.

A second related empirical assumption in the MS is that all evolutionary change is gradual. Harking back to Darwin's own work (1958), it is assumed that evolutionary change occurs in incremental steps, and that the size of those steps is small (Mayr and Provine 1998; Huxley 2010). ${ }^{3}$ This assumption responds to the second part of the origination problem-explaining how evolution can come about; it explains how complex adaptation can arise (via the accumulation of small adaptive steps).

Advocates of the EvoDevo alternative question the empirical justification for these assumptions of the MS, and thus whether the MS presents an adequate response to the origination problem (Müller and Newman 2005). They offer two sources of evidence motivating their skepticism.

\footnotetext{
${ }^{1}$ While the origination problem was identified and discussed both by Darwin and the architects of the MS, it was not considered a major challenge, and, by some, even considered to be resolved (Burian 1988). The structural alternative, while always present, has only recently gained prominence through EvoDevo (Amundson 2005).

${ }^{2}$ It is important to keep in mind that both the assumptions discussed here are simplifying assumptions (idealizations) rather than empirical claims. While advocates of the MS defend a gene-centric picture of evolution, they do not deny that the supply of variation to natural selection is biased in some situations; rather they claim that such situations are the exception to the vast majority of circumstances.

${ }^{3}$ Note, this is not an assumption about the rate of evolutionary change; it rather is an assumption about the nature of that change.
}

First, advocates of EvoDevo offer evidence that the supply of phenotypic variation to natural selection is frequently biased and constrained, particularly by development. This evidence suggests that architects of the MS underestimated the depth of the origination problem; rather than being explanatorily impotent with respect to the outcomes of evolution, genetic recombination and mutation (and other processes, such as development) may be very important to accounting for the tree of life (Alberch 1982; Maynard Smith et al. 1985; Hall 1999; Jablonka and Lamb 2006; Müller 2008).

Second, proponents of EvoDevo offer evidence of traits that do not fit the gradualist picture presented in the MS. Features such as the tetrapod limb (Capdevila and Izpisúa 2000), the neural crest (Hall 2005), the shell of turtles (Gilbert et al. 2001; Rieppel 2001), and feathers (Brush 1996; Prum 1999; Prum and Brush 2002) are all examples of characters that appear discontinuous with the characters that evolved before them. They are apparent counterexamples to the idea that all evolutionary change is gradual, and thus purely the product of genetic variation as it is traditionally construed.

Such jumps in phenotypic space are difficult for the MS to account for mechanistically, further undermining the simplistic picture of origination it presents (i.e., it is difficult to explain these phenomena solely using selection coupled with random mutation and recombination). ${ }^{4}$ Random mutations of large phenotypic effect are unlikely to be fitness enhancing or neutral; they are much more likely to be deleterious, and thus unlikely to be maintained in populations under selection. Furthermore, even if by luck a trait generated by a mutation of large phenotypic effect were to turn out to be adaptive, it is unlikely that it would become fixed in the population where it arose, because fixation relies on inheritance and amplification. Both require persistence and reproduction but the persistence of "one-off" characters of any type in a population is highly vulnerable to chance events. Individuals that have a strong propensity to survive and reproduce do not always do so, making the fixation of adaptive mutations of large

\footnotetext{
${ }^{4}$ Not everyone agrees with this assessment of the explanatory power of the MS (e.g., Hoekstra and Coyne 2007; Lynch 2007a, b). They argue that the MS, and population genetics in particular, has the means to account for the origination of form via the basic mechanisms of genetic recombination and mutation, and that those who deny this are only able to do so because they assume an impoverished picture of the causal power of genetic drift and recombination. Rather than offering an unlikely explanation for "jumps in phenotype," small changes in the genotype, they claim, are capable of generating significant phenotypic shifts via their action in broader gene networks and on accumulated neutral mutations. While interesting, I set aside this challenge to the EvoDevo picture here. This issue will only be resolved by further research into novelties and innovation. Such research requires a clear notion of what is to be explained and thus, if anything, provides motivation for the work herein.
} 
phenotypic effect unlikely. Ultimately, while genetic mutation and recombination alone offer a potential explanation for "jumps" in phenotypic space, they offer a highly improbable one (Goldschmidt 1940).

The EvoDevo research program, in exploring the role that development plays in evolution, offers an alternative means of explaining the origination of traits and "gaps" in the tree of life to that offered in the MS. Advocates of EvoDevo take seriously the role that development can play in structuring the supply of phenotypic variation and facilitating the generation of stable phenotypes in the face of genetic change, thereby enabling evolutionary "jumps" in phenotypic space to take place (Wagner 2005, 2012). For example, they point to the role that plasticity in the development of the nerves within the limbs of mammals plays in the generation of a viable phenotype even when mutations that have significant effects on limb morphology occur (Kirschner and Gerhart 2006). Many in EvoDevo believe that discontinuities in the tree of life cannot arise without the action of special innovative mechanisms at the developmental level (e.g., Müller 2010). Although others are less strident in their a priori assumptions, ${ }^{5}$ a central focus in EvoDevo is upon gaining an understanding of how novelties or discontinuities in the tree of life arise, and the role development plays in this.

Despite agreement regarding the centrality of the problem of origination to the EvoDevo research agenda, there has remained long-standing disagreement about the appropriate terminology to use within this domain. One source of such disagreement has been the commonly used term "novelty" (and the associated term "innovation").

\section{Defining Novelty: The Problem}

The term "novelty" features heavily within discussions of origination in EvoDevo. Unfortunately, there is no clear agreement on what the term means. Table 1 is a summary of the key definitions of the term in the literature.

As shown in Table 1, there are multiple definitions of novelty in use within EvoDevo. Furthermore, these definitions differ enough to give conflicting assessments of novelty. For example, according to definitions (1), (2), and (5), whether or not a trait is a novelty depends on how it evolved. In contrast, definitions (3) and (4) are "forwardlooking" - whether or not a trait is a novelty depends on the ways it can change, or changes it can facilitate, in the future (Brigandt and Love 2012). As a result, a trait could feasibly count as a novelty according to (1) but not according to (3). To illustrate, although the appearance of a

\footnotetext{
$\overline{5}$ Hall (2005), for example, does not rule out the possibility that the type of changes that underpin standard phenotypic variation also underpin large phenotypic changes in at least some cases.
}

non-homologous trait, such as a new digit (a novelty on definition (1)), within a population will open up some parts of phenotypic possibility space to a population for future evolution, it is likely to restrict others (and thus, will not necessarily be a novelty on definition (3)). This is not the only conflict between the definitions. For example, Arthur's (2000) definition (5) is extremely permissiveany new variation that arises in a population counts as a novelty (no matter how discontinuous it is with the previous variation) - while definition (1) is quite restrictivenovelty only refers to traits that are clearly discontinuous with earlier variation. Hence, traits that are novelties according to (1) will represent only a subset of those that are novelties according to (5) (Pigliucci 2008).

In part, these conflicts arise because there are three broad types of novelty definition in play: process-based definitions, character-based definitions, and prospective-product definitions. According to process-based definitions, novelties are those traits generated by a particular process. Definition (2), for example, is a process-based definition. Critics of these types of definition argue that they fail to reliably pick out discontinuities in the tree of life of the type that cannot be explained by the MS; i.e., they fail to pick out the explanandum of interest to EvoDevo (Peterson and Müller 2012). In contrast, character-based definitions focus on character homologies (i.e., discontinuity in characters). Definitions (1), (5), and (6) are broadly character-based. Such definitions are criticized for being too restrictive (Moczek 2008; Pigliucci 2008), and too reliant on the already problematic concept of homology (Moczek 2008; Brigandt and Love 2010; Hallgrímsson et al. 2012; Peterson and Müller 2012). ${ }^{6}$ The third type of definition, prospective-product definitions, relates to the potential for the origination of a trait to allow future evolution. For example, definitions (3) and (4) focus on the impact that a trait has on the adaptive potential of a lineage. Such definitions are difficult to use as they rely on information about counterfactual possibilities. They also do not adequately distinguish between standard genetic variation and the type of phenotypic change of interest to EvoDevo, i.e., discontinuous change.

It should be unsurprising that there are many definitions of a key term such as novelty in a science as young as EvoDevo. Unfortunately, for my purpose here the many definitions have the potential to hinder any attempt to apply

\footnotetext{
${ }^{6}$ Müller (2010) offers a more nuanced account of novelty which responds to criticisms of Müller and Wagner's (1991) definition (also see discussion in Peterson and Müller 2012). While I am in favor of this more nuanced approach, it still relies on attribution of homology and the use of concepts such as "body plan" which are hard to conceive of in the behavioral domain. Thus, in this article I focus on the more rudimentary definition as a way to gain some traction on the issue (more nuanced definitions of behavioral novelty will inevitably follow).
} 
Table 1 Usages of the term "novelty" in evolutionary biology

\begin{tabular}{lc}
\hline The term "novelty" is used to refer to & Reference(s) \\
\hline $\begin{array}{l}\text { A new feature in a group of organisms that is not homologous to a feature in an } \\
\text { ancestral taxon }\end{array}$ & $\begin{array}{c}\text { Müller and Wagner (1991, p. 243), West-Eberhard (2003, } \\
\text { p. 98), Hall (2005, p. 549) } \\
\text { Hallgrímsson et al. (2012) }\end{array}$ \\
$\begin{array}{l}\text { Traits that "have evolved both by a transition between adaptive peaks on the } \\
\text { fitness landscape and ... overcome previous developmental constraint" }\end{array}$ & $\begin{array}{l}\text { Müller and Wagner (2003), Wagner and Stradler (2003), } \\
\text { A trait which allows for future morphological variation and diversification that did }\end{array}$ \\
$\begin{array}{l}\text { not previously exist (i.e., increases evolvability) } \\
\text { “...any newly acquired structure or property that permits the performance of a }\end{array}$ & $\begin{array}{c}\text { Mayr (1963, p. 602) } \\
\text { new function, which, in turn, will open a new adaptive zone" }\end{array}$ \\
$\begin{array}{l}\text { Apomorphies: unique derived features of taxa that carry no phylogenetic } \\
\text { information }\end{array}$ & $\begin{array}{c}\text { Arthur (2000, p. 811) } \\
\text { "New traits or behaviors, or novel combinations of previously existing traits or } \\
\text { behaviors, arising during the evolution of a lineage, and that perform a new }\end{array}$ \\
\begin{tabular}{l} 
function within the ecology of that lineage" \\
\hline
\end{tabular}
\end{tabular}

the EvoDevo conceptual framework to behavior. When applying a conceptual framework in a new domain it helps to have clear, agreed-upon concepts in the first place. Although the definitions in Table 1 are reasonably succinct (so confusion could be avoided by stipulation), conceptual disagreement is often a symptom of deeper theoretical divisions within a science. This makes deciding which definition of novelty to use in an EvoDevo of behavior less straightforward than if there were one clear and agreedupon approach. Brigandt and Love (2012) offer a way forward.

\section{Defining Novelty: A Solution}

Brigandt and Love (2012) use the persistence and utility of the many existing usages of the term novelty in EvoDevo to argue that the role of the concept in the field is epistemic rather than definitional. Novelty, they say, is an organizing concept for EvoDevo. The various novelty definitions in Table 1 structure the problem space in the field, clarifying various aspects of its explanatory agenda in different, but still useful, ways. In this light, the utility of each definition varies depending on the research question being entertained. For some questions, definition (1) will be best; for other questions, the other definitions might be more useful. Hence, according to Brigandt and Love (2012), those disagreeing about the "right" concept or definition of novelty are misguided, at least insofar as they are motivated by a desire for a single unified approach. No single unified concept can appropriately capture all the roles played by the term in EvoDevo; rather, "novelty" is best understood as referring to a cluster of related concepts and phenomena.

This approach to novelty captures well the role the various existing novelty definitions play in science. Traits that satisfy definition (3), for example, are a useful guide to the "special mechanisms" underpinning evolvability.
Similarly, non-homology (definition (1)) serves as a useful guide to researchers interested in "special" developmental mechanisms of "innovation." As discussed in the introduction, many in EvoDevo believe that these "special" mechanisms will be developmental, and thus novelty, whether defined as (1) or (3), offers a promising area of research for those seeking to establish the importance of development in evolutionary biology.

\section{Focusing on Novelty as Non-Homology}

Here, I will assume Brigandt and Love's (2012) cluster concept account, thereby justifying focusing on one particular definition of novelty-novelty as non-homology. ${ }^{7}$ I have chosen to focus specifically on novelty qua nonhomology for three reasons.

First, identifying novelty with non-homology is one of the oldest, and by far the most common, approaches within EvoDevo. Hence, it is likely to offer a useful theoretical kind-concepts that are not useful tend not to persist-and an uncontroversial starting point for work on novelty in behavior.

Second, there is an (albeit, small) empirical literature on homology in behavior that I can draw on in modifying this approach for my purposes here. For other existing definitions of novelty, the conceptual groundwork is not nearly so clear. For example, adopting definition (2) of novelty for an EvoDevo of behavior would be highly challenging in practice, as it relies heavily on empirical information about

\footnotetext{
${ }^{7}$ Broadly speaking, two traits are non-homologous if they are not derived from the same ancestral structure. Note that non-homology is not the same thing as homoplasy (resemblance due to common selective history) despite this also often being contrasted with homology.
} 
behavioral adaptation and development that is not yet readily available. ${ }^{8}$

Third, the non-homology approach to novelty allows us to target research in EvoDevo to traits whose evolution is likely to be significantly influenced by development (i.e., evolutionary events where we might expect mechanisms other than genetic mutation and recombination to be in play). For the reasons discussed earlier, non-homologies, as discontinuities in the tree of life, are traits that are most likely to originate through special mechanisms of "innovation." Hence, non-homology serves as a good heuristic for limiting the scope of enquiry in EvoDevo, and making tractable the challenge of explaining the origination of traits (Brigandt and Love 2012).

In accordance with Brigandt and Love (2012), a good definition of novelty is one that focuses attention on some important question within the purview of the origination problem. Attempts to arbitrate between the different candidate definitions of the term in EvoDevo on the grounds that some pick out "true" novelty and others do not are mistaken. Rather, we should ask ourselves for each candidate definition: "Does this definition of novelty pick out a class of entities that can play a useful role in the science of origination?"

Importantly, novelty as non-homology plays such a role even if it turns out that many apparent "novelties" or nonhomologies are actually homologous to earlier characters when explored further. The turtle shell offers an illustrative case in point here. Not long ago, the turtle shell was considered a paradigmatic example of a non-homologous trait (i.e., a novelty) (Rieppel 2001). Recently, however, fossils of species ancestral to turtles have been found that have bony structures related to the ribs located in the appropriate parts of the body plan for them to be the morphological precursors to the turtle shell. The turtle shell is derived from these existing morphological structures rather than a de novo structure in its own right, and thus is no longer considered a novelty (Hall and Kerney 2012). Novelty as non-homology has not failed here, however, looking for non-homologies has allowed researchers to focus their efforts upon a case that tests some of the key premises of EvoDevo relating to origination. Finding precursors to the turtle shell challenges the idea that there are discontinuities in the tree of life, and that developmental mechanisms are central to explaining them. If it turns out that all cases of apparent non-homology are explicable by the traditional mechanisms of variation and gradual change (as may be the

\footnotetext{
${ }^{8}$ It is also dependent on the use of the "adaptive landscape" model. Applications of this model within evolutionary biology have been both controversial and methodologically challenging (Gavrilets 2004; Pigliucci and Kaplan 2006; Kaplan 2008.
}

case for turtles), ${ }^{9}$ the EvoDevo challenge to the MS will likely fail. ${ }^{10}$ If, on the other hand, some cases of nonhomology hold up to scrutiny, they offer a good starting point for those looking for special developmental mechanisms of innovation and origination. Either way, novelty as non-homology will have served a useful purpose in organizing and structuring the research agenda of EvoDevo. It is in this light that the remainder of the article focuses on novelty as non-homology. In particular, I focus on whether the concept can be fruitfully applied to behavioral traits. As we shall see, the second conceptual challenge to an EvoDevo of behavior that I introduced at the start of this article-the focus on morphological features in our concepts-presents a barrier to this project (though not an insurmountable one). I begin by looking at the application of the non-homology concept to morphological traits in a little more detail.

\section{Identifying Non-Homology: The Morphological Tradition}

Non-homology is a relationship that holds between traits in related species which are not derived from the same ancestral structure. It is best understood when contrasted with homology (traits that share a common ancestral origin as a consequence of descent from a common ancestor). Determining whether traits are non-homologous (i.e., novelties) requires us to test perceived discontinuities in the tree of life, and establish whether they are real or merely illusory. Within EvoDevo the classification of traits as novelties qua nonhomologies thus involves ruling out the possibility that they are homologies. In effect, if a trait cannot be shown to be homologous to any other then it is considered to be a novelty (Müller and Wagner 1991; Müller and Newman 2005; Müller 2010). Unfortunately for our purposes here, homology is traditionally a strongly morphological notion that remains difficult to apply to behavior.

\section{Defining Homology}

The concept of homology originated with the famous Victorian naturalist Richard Owen (1843, p. 379), who

\footnotetext{
9 Although the evidence of ancestral shell precursors in the turtle shell case undermines its status as a novelty qua non-homology, the intermediaries are still not sufficient to show that evolution in this case has been gradual. It may be the case that there have still been "jumps" (albeit smaller ones) in phenotypic space here.

${ }^{10}$ Many would still argue that there was a role for EvoDevo even were gradualism to be vindicated; the EvoDevo research program offers a particular type of explanation not offered by the MS, i.e., lineage explanations (Calcott 2009). This type of mechanistic explanation is importantly different from the type of explanation that is offered by traditional evolutionary biology and population genetics.
} 


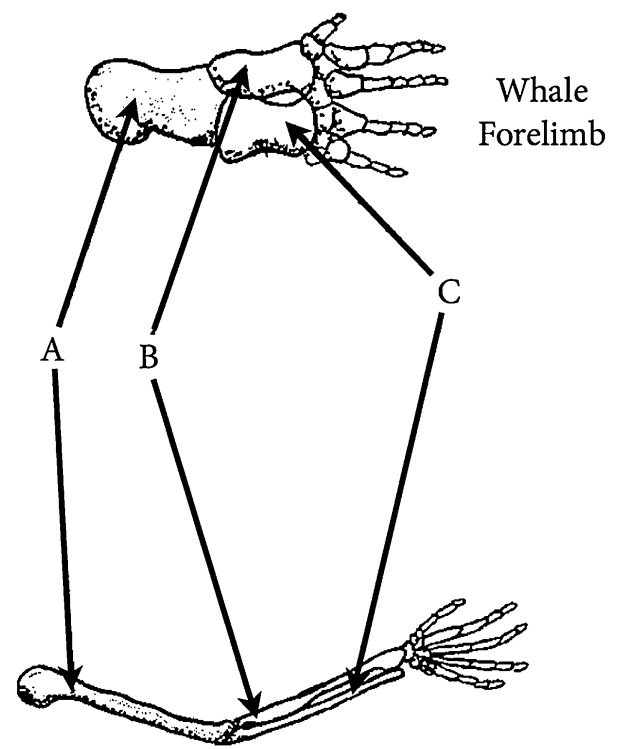

Human

Forelimb

Fig. 1 An example of homology defined using relative position. The forelimbs of the whale and human have the same structure, reflecting their shared ancestry. Homologous relationships between some of the bones are marked

defined it as "the same organ in different animals under every variety of form and function." He contrasted it with analogy or homoplasy, which he described as "a part or organ in one animal which has the same function as another part or organ in a different animal" (p. 374). Owen's approach to homology, motivated by the desire to identify the phylogenetic relationships between organisms, is fundamentally a morphological notion. He was concerned about comparing the presence and absence of "organs" and the functional role they play across species. Modern approaches to homology have remained relatively true to Owen's approach in practice (despite all agreeing that there are behavioral homologies). Three operational criteria, originating with Adolf Remane (1952), ${ }^{11}$ are each considered sufficient for homology today (Brigandt 2003; Griffiths 2007).

The first of these criteria concerns the relative position of the characters in the overall body plan of the organisms they are found in. It says that two bones are homologous if they share the same position in the body plan. For example, as illustrated in Fig. 1, when we compare the forelimbs of the whale and human, we see similar arrangements of bones, although the bones vary in size and shape. This similarity in the internal structure of the forelimb between whales and humans is the product of shared ancestry (both

\footnotetext{
11 The original 1952 text by Remane is in German. I relied on a summary from Griffiths (2007) in composing this work .
}

species descended from a common ancestor that had the basic bone arrangement), and hence they are homologous.

Remane's second criterion concerns the shared possession of "special qualities"-non-adaptive and distinctive features of the characters being considered for homology. As these features serve no function (and hence, are not the product of convergent evolution), when two organisms share special qualities they are more likely to share a common ancestor. The more complex and more distinctive these special qualities are, the greater the likelihood that their presence in two different species is due to common ancestry, rather than being independently derived. The most famous example of a special character is the positioning of the blood supply to the retina between the retina and the source of light in vertebrates. This positioning of the blood supply is common to all vertebrates and is not seen in invertebrate "eyes" such as those in cephalopods and arthropods. Furthermore, it is also not a functionally important feature of the vertebrate eye. For these reasons, the positioning of the blood supply in the vertebrate eye is considered to be a "special quality" for the purposes of identifying homologous relationships, and has been used as evidence of the common origin of all vertebrate eyes (Griffiths 1997, 2007).

Remane's third criterion concerns continuity between characters. Two characters, according to this criterion, can still be homologized (even if they fail the first two criteria) if they can be connected by a series of intermediary traits in other species. For example, there is a pair of bony structures at the rear end of the whale skeleton. If we trace the evolution of these bony structures using fossils and the comparison of whale anatomy with extant relatives such as the hippopotamus, we can see that they are actually vestigial hind limbs. Although they lack some elements of the typical tetrapod limb, the bone structures are actually homologous to the hind limb of mammal tetrapods.

\section{Using Remane's Criteria to Identify Non-Homology}

Remane's (1952) operational criteria were originally intended for use in discerning homologies from homoplasies-something required when establishing the phylogenetic proximity of species. Those in EvoDevo concerned with novelty and innovation, however, have also made use of these criteria in the reverse when identifying nonhomologies; in effect, novelty begins where homology ends (Moczek 2008). Müller and Wagner's (1991) definition of novelty makes this explicit. According to them, "a morphological novelty is a structure that is neither homologous to any structure in the ancestral species nor homonymous to any other structure in the same organism." In practice, this means that if a character found within a given lineage satisfies any of the above criteria for being 
homologous to some other trait in the lineage, they cannot also be non-homologous, and thus fail to be novelties. Hence, when a potential novelty is being considered, researchers look to the traits of close relatives for precursors or related characters.

If there is no ancestral character in the location of the potential novelty, there are no apparent intermediaries, and no shared special qualities between the focal trait and other traits in the lineage, then this is evidence for its nonhomology. For example, the appearance of a distinctly new articulated joint in the presence of all the other ancestral joints, and in the absence of any special characters would be a novelty. The recent research into the turtle shell discussed earlier offers a good example of how these criteria are used; current evidence being used to challenge the previously well-accepted view that the shell is a novelty (Hall and Kerney 2012).

\section{Identifying Non-Homology: The Behavioral Domain}

\section{Can We Use Remane's Criteria in an EvoDevo of Behavior?}

No one would deny that related species can share behavioral traits due to common ancestry. Indeed, Konrad Lorenz (1974) saw the identification of homologies and analogies as an essential source of knowledge in evolutionary biology, including the evolutionary biology of behavior. Unfortunately, however, it has historically been the view that the plasticity of behavioral traits undermines any attempts to identify behavioral homology at the general functional level (Schneirla 1957; Atz 1970; Hodos 1976; Beer 1984). In particular, the identification of homology and analogy in morphology has tended to rely on assumptions about phylogenetic proximity and resemblance to determine the identity of apparently homologous traits, but the employment of these methods in behavioral biology would rely on assumptions about the transformability of behavioral traits that are unjustified (Beer 1984). The plasticity of behavior has thus seen that attempts to homologize behavior thus far have largely been reductionistic in nature. These attempts have used the morphological structures that are involved in behaviors, rather than the behaviors themselves, as "tie breakers" in decisions about whether traits are homologues or not (Hall 2013). Although there have been attempts in the past 20 years to offer operational criteria for homology in behavior that are analogous to those offered by Remane (1952)—most notably by ethologist John Wenzel (1992)—-these criteria have not been terribly successful. Behavioral biologists have tended to rely on morphological data rather than behavioral information when considering claims about the evolutionary relationships between organisms. To some extent such skepticism about homologizing behavior is warranted.

In the past, the main motivation for the identification of homologies was to establish common ancestry when building phylogenetic trees. For such ends, traits that are less variable are more reliable sources of evidence than those that are very plastic (and thus may offer misleading evidence). In modern phylogenetics, for example, genetic similarities are now widely agreed to "trump" morphological data in the building of phylogenetic trees. There are numerous accounts in the scientific literature of newly uncovered molecular data causing the reconsideration of phylogenetic relationships established using traditional morphological techniques. This focus upon genetic similarities is due to the fact that morphological similarities between traits are possible despite lack of common ancestry. Genetic similarity without common ancestry is much less likely to occur. Thus, genetic similarity offers a better source of evidence of the phylogenetic relationships between species than similarity at the phenotypic level. In EvoDevo however, homology plays a different role.

As discussed earlier, researchers in EvoDevo are interested in whether an apparently new trait within a species is derived from some trait in an ancestor (i.e., is a homologue) or is novel (i.e., is not a homologue), rather than whether two species are related. It is also the case that, as non-homology is a heuristic for guiding research in EvoDevo, it need not always be accurate (i.e., false positives are acceptable). The narrow and reductionist approach to homology in phylogenetics is thus not appropriate for EvoDevo, and many in EvoDevo have advocated a pluralistic alternative.

In what follows, I outline in a little more detail this pluralistic approach to homology, highlighting its advantages for EvoDevo. Given the fresh light shed on the issue of homology in EvoDevo by the pluralistic picture, I then take Wenzel's (1992) operational criteria for behavioral homology and consider their utility for the study of novelty in an EvoDevo of behavior.

\section{Pluralism and Homology}

Patterns of continuity and discontinuity in phenotypes are not always accompanied by discontinuities in the associated developmental or genetic architectures (Moczek 2008). To illustrate, the eyes of vertebrates, cephalopods, and arthropods are remarkably similar morphological features in many ways. If we look at the tree of life in terms of morphology, however, we see big gaps between the cephalopod, arthropod, and vertebrate eyes-each type of eye arose as a novelty in its own lineage relatively independently. Interestingly, this discontinuity in morphology 
is not completely reflected in the relevant genetic structures. Key aspects of the development of the eye in all three taxa (in fact the entire Bilateria) are actually governed by the action of a common master gene, Pax6 (Bolker and Raff 1996; Rutishauser and Moline 2005). This gene represents an example of a deep homology in the genotype despite the presence of morphological discontinuity.

Similarly, genetic and developmental discontinuities have been identified that do not correspond to phenotypic discontinuities. For example, the segmentation of insect bodies is considered a homologous morphological feature despite the fact that the developmental mechanisms and genes governing segmentation vary significantly between species. This disjoint between the phenotype and genotype in insects exists because of the action of a number of developmental mechanisms that ensure the stable expression of the insect body plan despite underlying change in genetics and development (Abouheif 1997).

Variation in the patterns of discontinuity and continuity in characters across development, genotype, and phenotype are also seen in the behavioral domain (Lauder 1986, 1994; Wenzel 1992; Rendall and Di Fiore 2007; Hall 2013). For example, human language, sonar navigation in ocean mammals, and birdsong lack the type of phenotypic similarity usually associated with homology. They are generally considered to be independent phenotypic novelties in each lineage. They do share a genetic homology, however, all being underwritten by the function of the FoxP2 gene, which is a deeply conserved vertebrate novelty (Scharff and Petri 2011; Hall 2013).

Evidence of this type has led to a more pluralistic understanding of homology in EvoDevo which underscores the fact that novelties at the gross phenotypic level are not always accompanied by discontinuities in genetics or developmental program, and vice versa. ${ }^{12}$ Discontinuities exist in the genetic, developmental, morphological, functional, cognitive, and behavioral domains, but they do not necessarily neatly line up together. For this reason, none of these domains should have priority over any of the others in terms of the assessment of homology in EvoDevo; whether two traits are homologous depends on the domain of assessment, though it may often be informative to compare what is going on at different levels (Striedter and Northcutt 1991; Hall 1994). For the purposes of those in EvoDevo concerned with novelty qua non-homology, this pluralistic approach to homology not only fits better with the way that the world is organized, but also better serves

\footnotetext{
$\overline{12}$ It is worth noting that the literature in this area of EvoDevo has tended to talk about this as the "hierarchical" approach. I have tried to avoid this label because the use of compositional language here is misguided and misleading-genes are not "lower-level" components of traits, they are rather precursors of traits. The reductionism in question here is explanatory rather than ontological in nature.
}

the explanatory role of novelty in appropriately targeting research in this domain.

Pluralism and Novelty

A pluralistic approach to homology, and thus novelty qua non-homology, better reflects our explanatory interest. For example, discussions of evolvability are frequently concerned with the features of developmental systems that either limit or open up the possible phenotypic variation to populations. Traits that are novelties qua non-homologies are a good starting point from which to identify such features. In this sense, novelty qua non-homology is a guide to research in EvoDevo rather than a definitive indicator of something "deeper." Identifying novelties at either the developmental, genetic, or phenotypic level is a starting point for study rather than the ultimate goal of research. Once a discontinuity of interest has been identified, comparing what is happening in different aspects of the organism can help us to understand how the discontinuity comes about. For example, new accounts of the evolutionary process incorporating mechanisms such as genetic accommodation (West-Eberhard 2003) emphasize the role of plasticity in driving evolution. ${ }^{13}$ If such "plasticity-first" accounts of evolution are right, we should expect novel traits to originate in plasticity within the phenotype that is ultimately maintained and stabilized in populations via novel genes or developmental mechanisms. A pluralistic approach to non-homology captures the important role of plasticity, while a purely morphological or developmental approach would fail.

Another benefit of the pluralistic approach to novelty is that it allows us to be agnostic toward the underlying mechanisms of innovation. This is helpful when trying to uncover the nature of innovation. For example, if we were to focus only on novelties in morphology we could quite easily miss the important role played by the accumulation of neutral mutations in the generation of many phenotypic novelties. An important insight of EvoDevo has been that two traits that are homologous morphologically may nonetheless be non-homologous genetically because of neutral genetic change in one species but not another (Wagner 2005). Hence, explaining a novel morphological feature very often requires reference to changes in genetics, development, and the environment that have occurred over

\footnotetext{
$\overline{13}$ There are a variety of related "plasticity-first" accounts of evolution in the literature including genetic accommodation, genetic assimilation, and the Baldwin effect. They originate early in the 20th century with Spalding (1873) and Baldwin (1896), amongst others (Lloyd Morgan 1896; Osborn 1896) but have reappeared many times (e.g., Simpson 1953, Waddington 1953a, b) before their current modern incarnation, which is best represented by the work of WestEberhard (2003).
} 
a period of time, rather than right when the new morphological feature is apparent. A pluralistic approach to novelty does not miss this important phenomenon. Having made clear novelty as it is understood with respect to morphological traits, let us now turn to behavioral traits.

\section{Wenzel: Remane Reconsidered}

While it is accepted within EvoDevo that novelty exists in behavior, little work has thus far been undertaken concerning behavioral novelty. It is unsurprising therefore that I have been unable to find clear operational criteria for establishing novelty qua non-homology. It is in this light that I here focus on an existing criteria for behavioral homology and co-opt them for use in an EvoDevo of behavior.

As already discussed, Remane (1952) proposed three operational criteria for identifying homologies (physical position, special qualities, and connection to intermediaries) and Wenzel (1992) offers behavioral equivalents for these along with examples from the literature. As with Remane's (1952) original criteria, a pair of traits need only satisfy one of the criteria to count as homologues. The criteria are as follows:

(1) Topology and positioning. A behavioral trait is homologous to some other behavioral trait if it occurs in the same position in a general pattern of behavior. Examples offered include the tail wagging movements of two species of cichlid fish that occur in the same place in courtship ceremonies (Baerends 1958), and the various phases of behavior in the greeting ceremonies of gulls and kittiwakes (Tinbergen 1959).

(2) Special qualities. Two behavioral traits are more likely to be homologous if they are complex and distinctive, and also if there are arbitrary (i.e., non-adaptive) similarities between the behaviors. Thus, behavioral traits that are complex, distinctive, arbitrary, and shared between two species are often classed as homologies. The examples offered include elements of bird displays and aspects of the webs of spiders.

(3) Continuity of intermediates. Two behavioral traits are homologous if we can trace a series of evolutionary steps between them on a continuum. Wenzel notes that this criterion is often hard to accurately assess because we do not have a clear notion of what the intermediaries in behavior would be (e.g., what would an intermediate function look like?). Sometimes living relatives will offer this information, but not always. As will be apparent, this problem recurs for non-homology.
Having laid out Wenzel's (1992) criterion, I now consider whether they can be used to identify behavioural novelty qua non homology in a manner similar to the way Remane's (1952) original criteria have been used to identify morphological novelties.

\section{Applicability of the Criteria for Identifying Non- Homologies in Behavior}

\section{Using the Criteria}

Using Wenzel's (1992) criteria we can compare behavioral traits within lineages, and establish whether they are homologous or not. If a behavioral trait in a given species fails to be homologous with any behaviors observed in ancestral or closely related species, then we can say that it is non-homologous or novel. In the case of topology and positioning, if we cannot find a behavioral element that occupies the same position or topology as the focal behavior in any closely related or ancestral species, then it is a non-homology or novelty. For special characters, we are looking for distinctive, non-adaptive elements that can help to identify earlier iterations of the focal character. If a special character is shared between a potential novelty and some ancestral behavior then it is a homology, not a novelty. Continuity can be used similarly to identify if a supposed novelty is derived from ancestral traits. If none of the criteria for homology are satisfied, we can assume the behavior is non-homologous (and, hence, a novelty). In the following, I offer two examples of how the criteria could be used in courtship displays and birdsong.

Courtship displays are highly ritualized sequenced display behaviors used to attract and impress potential mates. They are very common in birds, but are seen right across the animal kingdom. Importantly, these displays very often involve a series of highly stylized movements that are largely innate; this makes for a more straightforward analysis than in cases where behavior is highly flexible. The literature offers a clear example of Wenzel's (1992) criteria being used for homology and non-homology (albeit implicitly) in a series of papers on the courtship displays of the birds of paradise by Scholes (2008a, b).

Birds of paradise are a type of lek-breeding passerine that are most well known for their eccentric courtship displays and impressive plumage. The clade is also impressive for the disparity in courtship displays across the species within it-there are large gaps in the courtship display phenotypes between even closely related lineages. Scholes $(2008 \mathrm{a}, \mathrm{b})$ captures this discontinuity by comparing the elements and sequential ordering of elements in the courtship displays of various birds of paradise. He uses 
both topology and positioning, as well as functional distinctiveness, to discern whether the courtship display elements are homologous or novel.

To do this, Scholes (2008a, b) breaks up the displays into phases according to the particular function of the element (e.g., searching for mates, sampling those present, solicitation of individuals), and the order in which the elements occur. Scholes identifies novel characters that arise in the courtship displays across the clade by looking at the sequence of elements of each display, their function, and order. An element is a novelty if it serves a distinctively new function and/or occupies a novel position in the display sequence. For example, imagine a display sequence of $<$ searching for mates $><$ sampling females $>$ $<$ solicitation $>$. A novel behavioral element is identifiable by the presence of a functional element in a new position (e.g., $<$ searching for mates $><$ sampling females $>$ $<$ solicitation $><$ sampling females $>$ ), or a totally new functional element (e.g., $<$ searching for mates $><$ sampling females $><$ defensive display $><$ solicitation $>$ ).

This analysis picks out traits of interest to EvoDevo biologists. Indeed, Scholes uses his analysis of novelty in the courtship displays of the birds of paradise to argue that the disparity and diversity of courtship displays in the clade are not just a product of variation on existing display elements, but also the innovation of new elements. This is exactly the type of phenomena-disparity and diversity in the tree of life-that EvoDevo seeks to account for and the type of explanation that it seeks to offer.

It is worth noting before I move on that although I have focused on courtship displays here, there are a number of similar ritualized sequences of behavior within the animal kingdom that are likely to be similarly amenable to this analysis. Ritualized contest behavior (such as is seen in elks, moose, and other deer species), greeting behaviors (such as the sexualized greetings common in primate troops), feeding behaviors (such as the begging of baby birds), and other systematic and invariant behaviors, such as web building in spiders, are likely to be able to be analyzed using Wenzel's (1992) criteria.

Birdsongs consist of a stereotyped pattern of a number of repeated distinct syllable types. In all species of songbird studied, the particular songs a bird sings are learned from other birds during a sensitive developmental period (Slater and Lachlan 2003). Much like with courtship displays, birdsongs can be broken up into elements.

The largest unit of behavior is the repertoire; this refers to all the songs of a particular bird. This can be broken into smaller units at the level of songs-discrete segments of the repertoire that are made up of even smaller units known as song elements. Song elements are present in each song in particular order.
Variation at the level of the song arises in a number of ways, most often through the reuse of song elements from other parts of the repertoire, although occasionally through wholesale invention (Lachlan and Servedio 2004). The criteria for homology can be used to discern the former class of cases (which may be best classed as homologies) from the latter, which are discontinuous with earlier song elements, and thus non-homologous. In contrast to the case of courtship displays above, topology and positioning is not obviously useful here because of the ubiquity of reused elements. Rather, looking for a lack of intermediaries and lack of special qualities is more important.

Using sound analysis equipment, songs can be recorded and compared between individuals and across species to quantitatively assess the distinctiveness of song elements. If a song contains no existing song elements from a repertoire or no derivatives of existing elements, then it can be classed as a novelty.

Being able to identify novel song elements, and thus investigate the mechanisms of song innovation, has ramifications for our understanding of the evolution of birdsongs and songbirds more generally. There is evidence that song learning reduces the time to speciation in songbirds by increasing the rate at which new elements can propagate through populations (Lachlan and Servedio 2004). Given this, we might also expect song innovation to increase the rate of speciation by offering more grist for the cultural mill, so to speak. In other words, as with ritualized displays, we would expect lineages or clades with higher rates of song innovation to be bushier than those that have lower rates of song innovation. Explaining this diversity would require reference to phenotypic novelty, and the processes of innovation underpinning it.

\section{Challenges to Application of the Criteria}

There are three key challenges to the use of Wenzel's (1992) criteria in the behavioral domain. First, the use of the criteria relies on a clear means of delineating behavioral units. There is no principled means of delineating behavioral units in the literature (Martin and Bateson 2007, pp. 121-134). Second, the criteria-in particular the second and third-require a means of quantifying behavioral similarity and difference. As with the method of delineating behavioral units, measuring similarity and difference in behavior is notoriously difficult (Martin and Bateson 2007, pp. 121-134). Third, the criteria are difficult to apply to plastic traits. I will consider these each in turn.

\section{Delineating Behavior into Traits}

This challenge concerns how we divide behaviors and other related characters into elements. For example, our 
assessment of the novelty of an element of a bird courtship display using positioning is dependent on how fine or coarse-grained our division of the display into phases is. If we are coarse-grained in our delineation of the display into phases we may decide that, rather than being an entirely new element, a novel wing flap in a bird of paradise courtship display is best classed as variation on an existing element (much like a new fossa on an existing bone). If, on the other hand, we are more fine-grained in our delineation, we may decide that the same wing flap element is a novelty. Unlike in the case of a bony appendage-where we can visually discern between novel elements and mere flourishes by considering disconnections and articulations-it is not clear how to objectively segment a courtship display into elements, and hence, whether the apparently new element is an element at all.

The same problem recurs with both the special quality and presence of intermediates criteria. If we are coarsegrained in our delineation of behavioral characters it is very easy to get homology; if we are fine-grained novelty abounds.

While this challenge to the use of Wenzel's (1992) criteria is significant, it is a challenge faced by all studies of behavioral evolution; the problem of the appropriate unit of analysis for behavioral characters being a long recognized issue in ethology (Barlow 1968; Drummond 1981). It is tempting to respond, at least with respect to novelty, by simply saying that it doesn't matter whether our assessments of novelty are hard and fast or not as it is merely a guide to research, but this by itself is not a completely adequate response. Our criteria for novelty will not be useful in guiding research if they are so permissive as to make every variation in behavior a novelty, or so rigid as to make nothing novel.

Existing evidence from phylogenetics offers a potential solution to the problem. Phylogenies will already exist for many of the species whose behavior we wish to consider from the EvoDevo perspective. These phylogenies-built largely using data regarding morphological and genetic homologies offer the means to test the accuracy of any given delineation of behavior into elements. Such testing would involve, first, segmenting our behavior of interest into characters and elements using whichever candidate schema seems most appropriate. Second, identifying characters within a group of species as homologous or nonhomologous using Wenzel's (1992) criteria. Third, building a phylogeny of the group using this information. Fourth, comparing the new phylogeny we have developed using behavioral information with existing phylogenies. If the phylogenies match (i.e., the phylogenetic relationships represented in each tree are similar), then we have good reason to think that the segmentation scheme we have adopted identified the behavioral characters and their elements and their relationships accurately. If, however, the trees differ significantly, we should be skeptical about the adequacy of our segmentation technique. Phylogenetic data may also help with a second problem for applying the proposed criteria for non-homology.

\section{Assessing Behavioral Distinctiveness}

Both the criteria of special quality and of the presence of intermediates require us to assess the similarity and difference between characters; in other words, their distinctiveness. For behavioral traits this is challenging; first, because of the difficulties of appropriately delineating behavioral traits as above. Second, because it is hard to determine what the appropriate metric for assessing behavioral similarity should be. This problem is nicely illustrated using birdsong. While we can use software to analyze sound and consider its similarities and differences, what level of difference is appropriate for novelty is unclear. Is a slightly exaggerated trill on a song element sufficient for that element to be a novelty, for example? What if the trill was accompanied by an increase in pitch?

There is also good reason to think that we are not necessarily looking for distinctiveness that is absolute when looking for behavioral novelties. Many behavioral elements paradigmatically described as "innovations" in the animal behavior literature involve some level of redeployment. For example, the milk bottle opening by blue tits (Parus caeruleus) is often thought to be an impressive and interesting example of a behavior that is novel (Sherry and Galef 1984, 1990; Lefebvre and Bouchard 2003), but its distinctiveness is relatively low. The milk bottle opening behavior involves the redeployment of already used motor patterns (pecking) on a new medium (bottle caps). The behavior is not strictly functionally distinct (although we can make it so if we offer a very narrow account of what the function is) as pecking open parcels of food is normal blue tit behavior.

Once again, as with the delineation of behaviors, I suspect that the solution to this problem is to do some empirical work where we use multiple metrics for distinctiveness, and then assess which metrics guide us to interesting cases in the context of innovation in EvoDevo. We may find the methodology of using existing phylogenies to test the relationships we discover via behavioral methods discussed earlier useful here as well. Ultimately, however, whether or not to classify the types of "innovative" behaviors such as the blue tit bottle opening as novelties really depends on whether the metric we use which allows for their inclusion offers up a category of interest to EvoDevo. We can only work this out through empirical study. 


\section{Identifying Novelty When There is Plasticity}

The plasticity of behavioral traits can make the identification of novelty particularly difficult. Chimpanzees (Pan troglodytes) offer a good illustration of this. Chimpanzee groups exhibit a high level of within-population behavioral variability. This variation is primarily thought to be the product of differences in learning, innovation, and social transmission between chimpanzee groups rather than a consequence of inherited genetic differences. In short, the variation in behavior is a product of behavioral plasticity coupled with cultural inheritance (Whiten et al. 1999). In the case of chimpanzee behavior, Wenzel's (1992) criteria offer little assistance in the identification of novelty. First, there is often no species-specific behavior to compare any putative novelty to, which makes identifying a comparison class for novelty difficult and potentially arbitrary. And second, because chimpanzee behavioral traits can be transferred between individuals via learning, they can be inherited both vertically and horizontally, and thus, the continuity of intermediates criterion can present us with false negatives and positives. For example, an individual may have behaviors that their parents lacked but those behaviors should not be classed as novel because they were learned from nonrelatives (i.e., already present in the population). A third reason to be skeptical about the applicability of Wenzel's criteria in the chimpanzee case is that often in chimpanzee bands we can trace conformity in behavior to common learning rules or dispositions rather than a shared common ancestor. For example, many individuals within a band of chimpanzees may independently come up with the same behavioral solution to a foraging problem as a consequence of their use of similar learning rules. This muddies the water with respect to what is, and is not, a homologous behavior.

These challenges to applying Wenzel's criteria generalize to many more cases than just chimpanzees. According to recent analyses of intraspecific behavioral variability ("animal personalities"; Dall et al. 2004; Dingemanse and Wolf 2010; Réale et al. 2010; Stamps and Groothuis 2010) there are often significant variations in behavior within animal groups. Similarly, there is evidence of learning and the transmission of behaviors between individuals via social learning in many species (Avital and Jablonka 2000; Laland and Galef 2009). This may mean that the criteria for novelty offered here are only applicable to a small domain of behaviors rather than behavior in general. Regardless, this issue will not be resolved without further conceptual work.

\section{Conclusion}

I began the article by outlining why EvoDevo cares about discontinuities in the tree of life-such discontinuities are difficult to explain without reference to development. I then outlined criteria used in EvoDevo for identifying morphological novelty. Building upon modifications to these criteria for behavior by Wenzel (1992), I then offered the means to identify behavioral novelty. This article is a starting point for the exploration of behavioral novelty and innovation from the EvoDevo perspective. While I have offered a set of criteria that can be used to assess behavioral non-homology, there remain difficulties associated with their use. In this light, and given the role novelty plays in EvoDevo more broadly, the criteria I offer here are best understood as a heuristic through which we can focus our research into behavioral novelties and the origination of behavioral traits. In particular, the criteria allow us to objectively identify features that are candidate novelties. As with the turtle shell case discussed earlier, it may be that further study of cognition and development will ultimately offer a way to explicate these behavioral novelties within the paradigm offered by the MS, but this is not a problem for the criteria. Their role is to give focus for enquiry of those concerned with the origination of behavior. The criteria offer a place from which we can begin to understand the processes of behavioral innovation.

Acknowledgments Thank you to Brett Calcott, Werner Callebaut, Gerd Müller, and Kim Sterelny for their generous and insightful suggestions on this manuscript. Thank you also to the Sydney-ANU Philosophy Group and an anonymous examiner for their comments on earlier incarnations of this work. Financial support for this research was provided by the Australian National University and the Rotman Institute for Philosophy at Western University.

\section{References}

Abouheif E (1997) Developmental genetics and homology: a hierarchical approach. Trends Ecol Evol 12:405-408

Alberch P (1982) Developmental constraints in evolutionary processes. Evol Dev 3:313-326

Amundson R (2005) The changing role of the embryo in evolutionary thought: the roots of evo-devo. Cambridge University Press, Cambridge

Arthur W (2000) The origin of animal body plans: a study in evolutionary developmental biology. Cambridge University Press, Cambridge

Atz JW (1970) The application of the idea of homology to behavior. In: Aronson LR, Tobach E, Lehrman DS, Rosenblatt JS (eds) Development and the evolution of behavior. Essays in memory of T. C. Schneirla. Freeman, San Francisco, pp 53-74

Avital E, Jablonka E (2000) Animal traditions: behavioural inheritance in evolution. Cambridge University Press, Cambridge

Baerends GP (1958) Comparative methods and the concept of homology in the study of behavior. Arch Neerland Zool Suppl 13:401-417

Baldwin JM (1896) A new factor in evolution. Am Nat 30(441-51):536-553

Barlow GW (1968) Ethological units of behavior. In: Ingle D (ed) The central nervous system and fish behavior. University of Chicago Press, Chicago, pp 217-237 
Beer CG (1984) Homology, analogy, and ethology. Hum Dev 27:287-308

Bertossa RC (2011) Morphology and behaviour: functional links in development and evolution. Phil Trans R Soc Lond B 366:2056-2068

Bolker JA, Raff RA (1996) Developmental genetics and traditional homology. BioEssays 18:489-494

Brigandt I (2003) Homology in comparative, molecular, and evolutionary developmental biology: the radiation of a concept. J Exp Zool B (Mol Dev Evol) 299:9-17

Brigandt I (2007) Typology now: homology and developmental constraints explain evolvability. Biol Philos 22:709-725

Brigandt I, Love AC (2010) Evolutionary novelty and the Evo-devo synthesis: field notes. Evol Biol 37:7

Brigandt I, Love AC (2012) Conceptualizing evolutionary novelty: moving beyond definitional debates. J Exp Zool B (Mol Dev Evol) 318:417-427

Brown RL (2014) Rethinking behavioral evolution. In: Barker G, Desjardins E, Pearce T (eds) Entangled life: organism and environment in the biological and social sciences. Springer, Berlin, pp 237-260

Brush AH (1996) On the origin of feathers. J Evol Biol 9:131142

Burian RM (1988) Challenges to the evolutionary synthesis. Evol Biol 23:247-269

Calcott B (2009) Lineage explanations: explaining how biological mechanisms change. Br J Philos Sci 60:51-78

Capdevila J, Izpisúa BJC (2000) Perspectives on the evolutionary origin of tetrapod limbs. J Exp Zool 288:287-303

Dall SRX, Houston AI, McNamara JM (2004) The behavioural ecology of personality: consistent individual differences from an adaptive perspective. Ecol Lett 7:734-739

Darwin C (1958) The origin of species. Signet Classics, New York. Orig. 1859

Dingemanse NJ, Wolf M (2010) Recent models for adaptive personality differences: a review. Phil Trans R Soc Lond B 365:3947-3958

Dobzhansky T (1957) On methods of evolutionary biology and anthropology. Am Sci 45:381-392

Dobzhansky T (1971) Genetics of the evolutionary process. Columbia University Press, New York

Drummond H (1981) The nature and description of behavior patterns. In: Bateson PPG, Klopfer FH (eds) Perspectives in ethology, vol 4. Plenum Press, New York, pp 1-33

Gavrilets S (2004) Fitness landscapes and the origin of species. Princeton University Press, Princeton

Gilbert SF, Loredo GA, Brukman A, Burke AC (2001) Morphogenesis of the turtle shell: the development of a novel structure in tetrapod evolution. Evol Dev 3:47-58

Goldschmidt R (1940) The material basis of evolution. Yale University Press, New Haven

Griffiths PE (1997) What emotions really are: the problem of psychological categories. University of Chicago Press, Chicago

Griffiths PE (2007) The phenomena of homology. Biol Philos 22:643-658

Hall BK (ed) (1994) Homology: the hierarchical basis of comparative biology. Academic Press, San Diego

Hall BK (1999) Evolutionary developmental biology, 2nd edn. Kluwer, Dordrecht

Hall BK (2005) Consideration of the neural crest and its skeletal derivatives in the context of novelty/innovation. J Exp Zool B (Mol Dev Evol) 304:548-557

Hall BK (2013) Homology, homoplasy, novelty, and behavior. Dev Psychobiol 55:4-12

Hall BK, Kerney R (2012) Levels of biological organization and the origins of novelty. J Exp Zool B (Mol Dev Evol) 318:428-437
Hallgrímsson B, Jamniczky HA, Young NM, Rolian C, Schmidt-Ott U, Marcucio RS (2012) The generation of variation and the developmental bias for evolutionary novelty. J Exp Zool B (Mol Dev Evol) 318:501-517

Hodos W (1976) The concept of homology and the evolution of behavior. In: Masterton RB, Hodos W, Jerison H (eds) Evolution, brain, and behavior: persistent problems. Erlbaum, Hillsdale, pp 152-167

Hoekstra HE, Coyne JA (2007) The locus of evolution: Evo Devo and the genetics of adaptation. Evolution 61:995-1016

Huxley JS (2010) Evolution: the modern synthesis. MIT Press, Cambridge, MA. Orig. 1942

Jablonka E, Lamb MJ (2006) Evolution in four dimensions: genetic, epigenetic, behavioral, and symbolic variation in the history of life. MIT Press, Cambridge, MA

Johnston CE, Page LM (1992) The evolution of complex reproductive strategies in North American minnows (Cyprinidae). In: Mayden RL (ed) Systematics, historical ecology, and North American freshwater fishes. Stanford University Press, Stanford, pp 600-621

Kaplan J (2008) The end of the adaptive landscape metaphor? Biol Philos 23:625-638

Kennedy M, Spencer HG, Gray RD (1996) Hop, step and gape: do social displays of the Pelecaniformes reflect phylogeny? Anim Behav 51:273-291

Kirschner MW, Gerhart JC (2006) The plausibility of life: resolving Darwin's dilemma. Yale University Press, New Haven

Lachlan RF, Servedio MR (2004) Song learning accelerates allopatric speciation. Evolution 58:2049-2063

Laland KN, Galef BG (2009) The question of animal culture. Harvard University Press, Cambridge

Lauder GV (1986) Homology, analogy, and the evolution of behavior. In: Nitecki MH, Kitchell JA (eds) Evolution of animal behavior. Oxford University Press, New York, pp 9-40

Lauder GV (1994) Homology, form, and function. In: Hall BK (ed) Homology: the hierarchical basis of comparative biology. Academic Press, New York, pp 151-196

Lefebvre L, Bouchard J (2003) Social learning about food in birds. In: Fragaszy DM, Perry S (eds) The biology of traditions: models and evidence. Cambridge University Press, Cambridge, pp 94-126

Lloyd Morgan C (1896) On modification and variation. Science $4: 733-740$

Lorenz K (1974) Analogy as a source of knowledge. Science 185:229-234

Lynch M (2007a) The frailty of adaptive hypotheses for the origins of organismal complexity. Proc Natl Acad Sci USA 104(Suppl 1):8597-8604

Lynch M (2007b) The evolution of genetic networks by non-adaptive processes. Nat Rev Gen 8:803-813

Martin P, Bateson P (2007) Measuring behaviour: an introductory guide. Cambridge University Press, Cambridge

Maynard Smith J, Burian R, Kauffman S, Alberch P, Campbell J, Goodwin B, Lande R, Raup D, Wolpert L (1985) Developmental constraints and evolution: a perspective from the mountain lake conference on development and evolution. Quart Rev Biol 60:265-287

Mayr E (1963) Animal species and evolution. Harvard University Press, Cambridge

Mayr E, Provine WB (1981) The Evolutionary Synthesis. Bull Am Acad Arts Sci 34:17-32

Mayr E, Provine WB (1998) The evolutionary synthesis: perspectives on the unification of biology. Harvard University Press, Cambridge, MA

McLennan DA, Brooks DR, McPhail JD (1988) The benefits of communication between comparative ethology and phylogenetic 
systematics: a case study using gasterosteid fishes. Can J Zool 66:2177-2190

Moczek AP (2008) On the origins of novelty in development and evolution. BioEssays 30:432-447

Müller GB (1990) Developmental mechanisms at the origin of morphological novelty: a side-effect hypothesis. In: Nitecki MH (ed) Evolutionary innovations. University of Chicago Press, Chicago, pp 99-130

Müller GB (2007) Evo-devo: extending the evolutionary synthesis. Nat Rev Genet 8:943-949

Müller GB (2008) Evo-devo as a discipline. In: Minelli A, Fusco G (eds) Evolving pathways: key themes in evolutionary developmental biology. Cambridge University Press, Cambridge, pp 3-29

Müller GB (2010) Epigenetic innovation. In: Pigliucci M, Müller GB (eds) Evolution: the extended synthesis. MIT Press, Cambridge, MA, pp 307-332

Müller GB, Newman SA (2005) The innovation triad: an EvoDevo agenda. J Exp Zool B (Mol Dev Evol) 304:487-503

Muller GB, Wagner GP (2003) Innovation. In: Hall BK, Olson WM (eds) Keywords and concepts in evolutionary developmental biology. Harvard University Press, Cambridge, pp 218-227

Müller GB, Wagner GP (1991) Novelty in evolution: restructuring the concept. Annu Rev Ecol Syst 22:229-256

Osborn HF (1896) Ontogenetic and phylogenetic variation. Science 4:786-789

Owen R (1843) Lectures on the comparative anatomy and physiology of the vertebrate animals. Longman, Brown, Green, and Longmans, London

Pigliucci M (2008) What, if anything, is an evolutionary novelty? Philos Sci 75:887-898

Pigliucci M, Kaplan JM (2006) Making sense of evolution: the conceptual foundations of evolutionary biology. University of Chicago Press, Chicago

Prum RO (1999) Development and evolutionary origin of feathers. J Exp Zool 285:291-306

Prum RO, Brush AH (2002) The evolutionary origin and diversification of feathers. Quart Rev Biol 77:261-295

Ramsey G, Bastian ML, van Schaik C (2007) Animal innovation defined and operationalized. Behav Brain Sci 30:393-407

Reader SM, Laland KN (2003) Animal innovation: an introduction. In: Reader SM, Laland KN (eds) Animal innovation. Oxford University Press, New York, pp 3-38

Réale D, Dingemanse NJ, Kazem AJN, Wright J (2010) Evolutionary and ecological approaches to the study of personality. Phil Trans R Soc Lond B 365:3937-3946

Remane A (1952) Die Grundlagen des natürlichen Systems, der vergleichenden Anatomie und der Phylogenetik. Otto Koeltz, Königsstein

Rendall D, Di Fiore A (2007) Homoplasy, homology, and the perceived special status of behavior in evolution. J Hum Evol $52: 504-521$

Rieppel O (2001) Turtles as hopeful monsters. BioEssays 23:987-991

Rutishauser R, Moline P (2005) Evo-devo and the search for homology ("sameness") in biological systems. Theory Biosci $124: 213-241$
Scharff C, Petri J (2011) Evo-devo, deep homology and FoxP2: implications for the evolution of speech and language. Phil Trans R Soc Lond B 366:2124-2140

Schneirla TC (1957) The concept of development in comparative psychology. In: Harris DB (ed) The concept of development. Jones Press, Minneapolis, pp 78-108

Scholes E (2008a) Structure and composition of the courtship phenotype in the bird of paradise Parotia lawesii (Aves: Paradisaeidae). Zoology 111:260-278

Scholes E (2008b) Evolution of the courtship phenotype in the bird of paradise genus Parotia (Aves: Paradisaeidae): homology, phylogeny, and modularity. Biol J Linn Soc 94:491-504

Sherry DF, Galef BG (1984) Cultural transmission without imitation: milk bottle opening by birds. Anim Behav 32:937-938

Sherry DF, Galef BG (1990) Social learning without imitation: more about milk bottle opening by birds. Anim Behav 40:987-989

Simpson GG (1953) The Baldwin effect. Evolution 19:110-117

Slater PJB, Lachlan RF (2003) Is innovation in bird song adaptive? In: Reader SM, Laland KN (eds) Animal innovation. Oxford University Press, Oxford, pp 117-135

Spalding DA (1873) Instinct with original observations on young animals. Macmillan's Mag 27:282-293

Stamps J, Groothuis TGG (2010) The development of animal personality: relevance, concepts and perspectives. Biol Rev 85:301-325

Sterelny K, Griffiths PE (1999) Sex and death: an introduction to philosophy of biology. University Of Chicago Press, Chicago

Striedter GF, Northcutt RG (1991) Biological hierarchies and the concept of homology. Brain Behav Evol 38:177-189

Tinbergen N (1959) Comparative studies of the behaviour of gulls (Laridae): a progress report. Behaviour 15:1-70

Van Tets GF (1965) A comparative study of some social communication patterns in the Pelecaniformes. Ornithol Monogr No $2: 1-88$

Waddington $\mathrm{CH}$ (1953a) Genetic assimilation of an acquired character. Evolution 7:118-126

Waddington CH (1953b) The 'Baldwin effect', 'genetic accommodation' and 'homeostasis.' Evolution 7:386-387

Wagner A (2005) Robustness, evolvability, and neutrality. FEBS Lett 579:1772-1778

Wagner A (2012) The role of randomness in Darwinian evolution. Philos Sci 79:95-119

Wagner GP, Larsson HCE (2006) Fins and limbs in the study of evolutionary novelties. In: Hall BK (ed) Fins into limbs: evolution, development, and transformation. University of Chicago Press, Chicago, pp 49-61

Wagner GP, Stadler PF (2003) Quasi-independence, homology and the unity of type: a topological theory of characters. J Theor Biol 220:505-527

Wenzel JW (1992) Behavioral homology and phylogeny. Annu Rev Ecol Syst 23:361-381

West-Eberhard MJ (2003) Developmental plasticity and evolution. Oxford University Press, New York

Whiten A, Goodall J, McGrew WC et al (1999) Cultures in chimpanzees. Nature 399:682-685 